\title{
S39. Neuroleptic drugs
}

Immune Parameter and Neuroleptic Treatment in Schizophrenia. N. Müller, M. Schwarz, B. Bondy, M. Ackenheil

Psychiatric Hospital, University of Munich, Nußbaumstr. 7, 80336 Munich, Germany

Findings like raised titers of autoantibodies, raised $\mathrm{CD}^{+}-\mathrm{B}$ cells, elevated levels of soluble interleukin 2-Receptors, reduced suppressor-cell activity, enhanced numbers of $\mathrm{CD}^{+}$-cells, and the presence of heat-shock protein antibodies support the view that an abnormal immune reactivity may play a role in a subgroup of about one third of the schizophrenic patients.

Moreover, abnormalities of the CSF like raised IgG content and a damage of the blood-CSF barrier are described in a subgroup of schizophrenics and correlations between raised $\lg G$ content and schizophrenic negative symptoms suggest a close relationship between CSF-IgG and negative symptoms in schizophrenic patients.

Several studies show, that treatment with neuroleptics does not influence the altered immune parameters in schizophrenic patients, although long-term treatment studies are lacking. On the other hand, the numbers of $\mathrm{CD}^{+}{ }^{+}$and $\mathrm{CD} 4^{+}$-cells seem to have a predictive value for the therapeutic response to neuroleptics in schizophrenia: patients with a low cell number before neuroleptic therapy show a better therapeutic outcome. A negative correlation of the cell number with the spiperone binding sites of lymphocytes in a small number of patients support that view, because the spiperone binding is also discussed to represent an outcome marker.

A predictive value for therapeutic response of neuroleptics is also reported for $\mathrm{N}$-CAM, a neural adhesion molecule: lower serum $\mathrm{N}$ CAM levels correspond with better therapeutic outcome. Since the validity of immune alterations in schizophrenia is discussed controversely when only single parameters are measured, the estimation of a combination or characteristic pattern of immune parameters might be more valid and reliable in order to define subgroups of schizophrenics with respect to the therapeutic outcome.

Therefore we replicated our analysis of the $\mathrm{CD}^{+}-$and $\mathrm{CD}^{+}$cells and additionally estimated the levels of N-CAM, I-CAM 1 and solkble IL2-Receptors in groups of schizophrenic responders and nonresponders to neuroleptic therapy in order to evaluate if the predictive value for the outcome of neuroleptic therapy is better in of a pattem of immune parameters than in single parameters.
SWITCHING TO RISPEKIDONE: OPTIMISING MEIHODS

Dr. C.K. Kirov, Professor R.M. Murray, Dr. L. Kifkin, London, UK; Dr. S. Feeney, Sittingbourne, UK; Ur. A.V.M. Hughson, Glasgow, UK; Dr. N. Savla, Essex, UK; Dr. R.V. Seth, London, UK; Dr. A.C. White, Dr. N.M.J, Kennedy, Birmingham, UK

Whilst conventional neuroleptics are effective in the treatment of positive symptons of schizophrenia, they have much less effect on the negative syniptoms. In addition, probably the most troublesome 1 initation to conventional neuroleptics relates to extrapyramidal side effects. Studies conducted as part of the international clinical research programme have consistently demonstrated the efficacy of risperidone in reducing the magnitude of both positive and negative symptoms in relapsing and non-relapsing patients. Placebo-controlled studies indicate that patients receiving risperidone at doses up to $10 \mathrm{mg}$ per day show a low incidence of EPS, not significantly different to that seen with placebo.

In general, antipsychotics bave not been systematically studied to devise treatment switching regimes from one neuroleptic therapy to another. This open study in 40 patients examines a number of empirically derived strategies, formulated by an international group of psychiatrists.

The switching technique involved an abrupt cessation of existing neuroleptic, initiation of risperidone at doses in 1 ine with its prescribing information, and a variable period of continuing and reducing anticholinergic if used previously.

Efficacy has been evaluated using the Krawiecka scale and Clinical Global Impression, and side effects assessed using the Simpson and Angus and Barnes Akathisia scales, and the "success" of the switching method is defined as symptom scores which show no consistent worsening following the swiching to risperidone monotherapy.

To date, for those patients completing the study so far, all have shown either a stable or an improved clinical status. Further conclusions drawn from the results of this study on optinised methods of switching to risperidone will be presented. 
TREATMENT RESISTANT SCHIZOPHRENIA: PRELIMINARY FINDINGS WITH RISPERIDONE

G. Mercer, Ms. C. Murray, A. Rogers, D.G. Cunningham Owens, E.C Johnstone

Royal Edinburgh Hospital, UK

Between $5-25 \%$ of patients with schizophrenia are commonly described as resistant to drug treatment. Levels of treatment resistance have been defined by Phillip May. Our study at the Royal Edinburgh Hospital aims to recruit over 50 patients from central Scotland who fall into May's categories 4,5 or 6 . Our trial is concerned with optimising both drug and psychosocial methods of care. Patients selected fulfil DSM III R and PSE 9 criteria for schizophrenia. They are randomised into three groups, one of which involves the use of risperidone. As the study is ongoing, the final results will be presented elsewhere.

At the end of the study all patients are given the opportunity of open treatment with risperidone. Our overall impressions of the drug's clinical efficacy in this group of difficult patients is favourable. By January 94 , of over 28 patients treated with risperidone with treatment durations up to 2 years, 16 have shown clinical improvement (CGI), 6 were unchanged and 6 worse. We use doses between 4 and $16 \mathrm{mg}$ daily for continuing treatment.

In some the improvement has been dramatic, comparable to the anecdotal accounts reported in this field with clozapine. Extrapyramidal effects have been mild and in three cases there has been marked improvement in dyskinesia and Parkinsonism scores.

In conclusion, post-study experience with risperidone in this group of patients with treatment-resistant schizophrenia is favourable. Doses remain in line with Data Sheet recommendations for general use in the psychoses and the levels of extrapyramidal side effects have been low.

Risperidone, a serotonin-dopamin-antagonist (SDA) in the treatment of psychoses with combined psychotic and depressive syndroms

\section{A Hillert, W Maier, H Wetzel, O Benkert}

Department of Psychiatry, University of Mainz, Untere Zahibacher Str. 8, D.55101 Mainz, Germany

Patients with combined psychotic and depressive syndroms, fulfilling the DSM-III-R criteria of a major depressive episode with psychotic features or a schizoaffektive disorder, depressed type, are usually treated with a combination of an antidepressant and a neuroleptic. This combined therapy has proven to be superior to a monotherapy with either a neuroleptic or an antidepressant.

Because risperidone (RIS) is a very potent serotonin $5-\mathrm{HT}_{2}$ receptor antagonist, concomittant antidepressant properties of this antipsychotic might be expected. Results of an open pilot study gave first evidence.

Ten patients with a combined psychotic and depressive syndrom were treated with RIS $(2-10 \mathrm{mg} / \mathrm{d})$ for six weeks. Weekly psychopathological evaluation using the Brief Psychiatric Rating Scale (BPRS) and the Bech Rafaelson Melancholia Scale (BRMS) showed a marked decrease of psychotic (BPRS total score, baseline versus endpoint: 55.6 6.5 (mean \pm SD) versus $31.3 \pm 12.8$ ) and depressive symtoms (BRMS: $26.8 \pm 5.4$ versus $11.1 \pm 13.2$ ). Generally, the tolerability of RIS was good. Moderate parkinsonoid was observed in only two patients.

A multicenter double-blind parallel group trial $(\mathrm{N}=130)$ comparing RIS versus the combination of haloperidol and amitriptylin is ongoing, in order to futher evaluate the therapeutic effects of RIS in the treatment of combined psychotic and depressive syndromes.
CLOZAPINE SERUM LEVELS AND SIDE EFFECTS DURING STEADY STATE TREATMENT OF SCHIZOPHRENIC PATIENTS

$R$ Rosenberg, OV Olesen, $K$ Thomsen, $P N$ Jensen, M Bysted, J Sorensen, NA Rasmussen, C Refshammer. J Christensen.

Institute for Basic Research in Psychiatry, Department of Biological Psychiatry, Psychiatric Hospital in Aarhus, Skovagervej 2, DK-8240 Risskov, Denmark

Serum clozapine (S-Cloza) and serum desmethyl-clozapine (S-Descloza) concentrations were measured in 30 chronic schizophrenic in- and out-patients. All patients were in steady state with respect to clozapine monotherapy and in a stable condition with respect to their psychotic illness. The mean \pm SD $24 \mathrm{~h}$ clozapine dose was $367 \pm 149 \mathrm{mg} / 24 \mathrm{~h}$ ( range 100-700). There was a weak positive correlation between doses and the BPRS total score $(r=0.44, p<0.05)$. The mean \pm SD S-Cloza was $1437 \pm 1198 \mathrm{nmol} / /$ (range 196-5581). The S-Cloza was linearly correlated to dose but with a high interindividual variation at equal doses, e.g. a factor eight at $400 \mathrm{mg} / 24 \mathrm{~h}$, but a low intraindividual variability of $10 \%$. The S-Descloza averaged $77 \%$ of the S-Cloza and was highly correlated to S-Cloza $(r=0.90 ; p<0.001)$. The S-Descloza/dose ratio increased with age and duration of treatment. The side-effects registered were EEG abnormalities $(81 \%)$, tachycardy $(73 \%)$, increased liver enzyme activities $(60 \%)$, orthostatic hypotension (17\%) and moderate lycocytosis $(17 \%)$. Only EEG changes were correlated to $S$-Cloza $(r=$ $0.43 ; p<0.05$ ). No side effects were correlated to S-Descloza, doses or the duration of treatment. The frequency of side effects was higher than in studies using lower mean doses indicating a correlation between doses or S-Cloza and the frequency of side effects. It is concluded that clozapine fulfils the criteria for therapeutic drug monitoring. TDM may contribute to find the lowest effective dose with the fewest possible side effects.

\section{TREATMENT RESISTANT SCHIZOPHRENIA IN ADOLESCENTS - RESULTS OF CLOZAPINE TREATMENT}

Eberhard Schulz and Helmut Remschmidt, Dept. of Child and Adolescent Psychiatry, Philipps-University, Hans-Sachs-Str. 6, 35033 Marburg (Germany)

Reported are the results from a prospective study including 40 schizophrenic adolescents diagnosed according to DSM-III-R criteria out of whom 20 patients have been treated with clozapine.

The investigations included the assessment of psychopathology by means of the BPRS (Brief Psychiatric Rating Scale) and the scales for the assessment of positive and negative symptoms (SAPS, SANS) and were performed with 6 week inervals during a one-year follow-up period. Further on, clozapine, clozapine-N-oxide and $\mathrm{N}$ desmethylclozapine were measured in serum by a sensitive high performance liquid chromatographic method (HPLC), recently developed by our group.

In addition, plasma dopamine (pDA), norepinephrine (pNE), epinephrine (pE), 3methoxy-4-hydroxyphenylglycol(pMHPG) and serum serotonin (5-HT) measurements were performed by HPLC.

The analysis of plasma catecholamines, pMHPG and serum serotonin concentrations demonstrated, that the clozapine treated group differed significantly from the patients receiving conventional neuroleptic drugs. Changes in serum serotonin levels revealed a close association with negative symptoms and pMHPG concentrations were linked to BPRS depression score. Finally, the implications of out findings for therapeutic drug monitoring and response prediction in adolescent schizophrenia will be discussed,

With regard to the problem of pre-treatment non-response and severe side-effects under conventional neuroleptics, clozapine must be considered as a drug which offers an enrichment of therapeutic interventions in treatment resistant adolescent schizophrenia. 
RISPERIDONE VERSUS ZUCLOPENTHIXOL IN THE TREATMENT OF ACUTE SCHIZOPHRENIC EPISODES MO Huttunen', Tapani Piepponen ${ }^{2}$

'Department of Psychiatry, University of Helsinki, Finland

${ }^{2}$ Janssen Pharma Oy, Espoo, Finland

A double-blind, randomized, multi-centre, parallel-group study was conducted in Finland to compare the efficacy and safety of risperidone with zuclopenthixol in patients with acute exacerbations of schizophrenia or schizophreniform disorder. Ninety-eight patients were randomly assigned to treatment with risperidone or zuclopenthixol, in variable doses, for six weeks. Efficacy was assessed throughout by the Positive and Negative Syndrome Scale (PANSS) for schizophrenia and Clinical Global Impression (CGI). Safety assesments inciuded the Extrapyramidal Symptom Rating Scale, UKU Side-Effect Rating Scale, vital signs, body weight and laboratory screening. The mean daily dose at endpoint was $8 \mathrm{mg}$ for risperidone and $38 \mathrm{mg}$ for zuclopenthixol. Risperidone showed superior effects, of borderline significance, on the area under the curve for the total PANSS score and rate of clinical response. The onset of action was significantly shorter with risperidone than with zuclopenthixol. Fewer patient experienced extrapyramidal symptoms with risperidone so that significantly fewer risperidone-treated patients required antiparkinson medication. The two treatment groups did not show any marked differences on the UKU Side-Effect Rating Scale, although some 'psychic' effects were less marked with risperidone. The results indicate that risperidone is at least as effective as zuclopenthixol for the treatment of acute schizophrenic episodes, and that its use shows a trend towards greater improvement in the overall severity of symptoms and a significantly faster onset of action. While the general tolerability of the two drugs was comparable, risperidone was associated with a lower incidence of extrapyramidal symptoms.
CURRENTS ON PHARMACOTHERAPY OF SCHIZOPHRENIA: A DOUBLE BLIND STUDY ON THERAPEUTIC EFFECTS OF ISULPIRIDE VS HALOPERIDOL IN ACUTELY RELAPSED CIIRONIC SCHIZOPHRENIC PATIENTS.

Sarteschi P. Barracchia E.* Bertolino A.* Bertolino Al *
Casacchia M.** Ciapparelli A. Core L.** Fiori $L^{* * *}$, Guazzelli $M$ Jaretti Sodano A.***, Pastore G.F.***, Starnini S Institute of Psychiatry, University of Pisa, via Roma 67, 1-56100 Pisa, * Bisceglie (BA), ** L'Aquila, *** Torino (Italy)

A double blind multicentric study was carried out in randomized sequencies on 100 acutely relapsed, hospitalized chronic schizophrenic patients in order to evaluate clinical efficacy and tolerability of a $600-800 \mathrm{mg}$ daily dose of 1 - Sulpiride vs $15-20 \mathrm{mg}$ of haloperidol on positive and negative symptoms evaluated by means of BPRS, SANS and SAPS scales at recruitment $(\mathrm{T} 0)$ and after $1,2,4$ weeks of treatment. The sample included 8 negative, 31 positive and 61 mixed patients, mean age $38.7 \pm 11.9$ years, illness duration $16.5 \pm 10.9$ years. No differences were detected between groups at $\mathrm{TO}$. Four patients treated with 1-Sulpiride and 9 with haloperido dropped-out. Both groups showed a progressive, highly significant decrease on BPRS and on SAPS and SANS scores which started earlier in 1-Sulpiride treated patients. Factor analysis results indicate that both drugs strongly affected SANS items; while the 1-Sulpiride effects seem to be more remarkable on SAPS items "Bizarre Behavior" and "Formal Thought Disorders" and the haloperidol on items "Allucination" and "Delusions". Good responders (> 50\% reduction of BPRS total score) were 6 for 1 Sulpiride and 4 for haloperidol, moderate responders (25-50\% of BPRS reduction) were respectively 19 and 18 and poor responders $(<25 \%$ of BPRS reduction) respectively 21 and 19 . Side effects were similar in both treatments; they induced treatment discontinuation in 2 cases in 1-Sulpiride group and in 7 cases in haloperidol group. Results of this investigation indicate that a $600-800 \mathrm{mg}$ daily dose of I-Sulpiride is as effective as haloperidol on negative and positive symptoms of acutely relapsed chronic schizophrenic patients and that it is better tolerated at least in terms of drop-outs 